\title{
Research on Multi-service Operation Optimization of Energy Storage Based on Battery Life Model
}

\author{
Zhou Jie ${ }^{1}$, Zhang Xinzhen ${ }^{2}$, Liu Li ${ }^{1}$, Jiang $\mathrm{Ke}^{3}$, Li Haibin ${ }^{1}$, Su Lin ${ }^{3}$ \\ ${ }^{1}$ Economic and Technological Research Institute, State Grid Jibei Electric Power Co., Ltd., Beijing , \\ China,100038 \\ ${ }^{2}$ Sichuan Energy Internet Research Institute Tsinghua University, Chengdu , China, 610213 \\ ${ }^{3}$ China Energy Engineering Group Jiansu Power Design Institute Co., Ltd, Jiangsu, China, 211106
}

Keywords: energy storage; operational strategy; frequency regulation; time-shift energy arbitrage; whole-life-cycle income model

\begin{abstract}
Poor economy is one of the key problems that restrict the development of user-side energy storage. So the user-side energy storage needs to provide multiple services to improve economy. In this paper, comprehensively considering time-shift energy arbitrage service and frequency regulation service, a whole-life-cycle income model of energy storage based on the relationship between charge/discharge rate and battery life is proposed to maximize the income of energy storage. The economic optimal operational strategy is also proposed. Based on this model and the data of PJM electricity market, the economy of various operational strategies is simulated. The results show that the optimal control strategy for energy storage to provide time-shift energy arbitrage service is to charge/discharge at $1.0 \mathrm{C}$, and the optimal control strategy for providing frequency regulation service is to charge/discharge at $5.38 \mathrm{C}$. Taking the $8000 \mathrm{~kW} / 1000 \mathrm{kWh}$ energy storage as an example, the benefit of energy storage providing time-shift energy arbitrage service changed from negative to positive after the optimized regulation strategy adopted, and the cost-benefit ratio of frequency regulation increased by $79.2 \%$.
\end{abstract}

\section{Introduction}

At present, the main application mode of energy storage is to provide time-shift energy arbitrage, but its low rate of return limits the development of the energy storage industry. Literature [1] established a cost model for the whole life cycle of energy storage, but the model only analyzed the cost structure in the whole life, and did not establish detailed cost models. Literature [2] proposed an investment optimization model for energy storage in the whole power system to evaluate the benefits, but its cost model is relatively simple. In 2016, H. Dagdougui et al. [3] proposed a storage capacity allocation model considering photovoltaic power generation, and simulated the analysis of the energy storage system's participation in reducing peak load. Lou Suhua et al. [4] proposed a variable life model of battery energy storage in 2015, considering the effect of depth of discharge on battery life, but did not consider the charge and discharge rates to the battery energy storage life.

Based on the battery cycle life model, considering factors such as charge/discharge rate and battery life, the whole-life-cycle income model is proposed to maximize energy storage income. The optimal operational strategy for time-shift energy arbitrage service and frequency regulation service is proposed. A case study is carried out based on this model and electricty price data to verify the effectiveness of the optimized operational strategy

\section{Battery cycle life model}

According to the research of Li Xinran et al. [5], the cycle life model considering the discharge depth was used in this paper. Based on the variable life model proposed by Lou Suhua et al. [4], the discharge electricty at different rates was converted into the equivalent discharge depth, and the battery cycle life model was constructed. 


$$
\begin{gathered}
\mathrm{n}=N_{r} *\left(\frac{D_{\mathrm{r}}}{D_{\mathrm{a}}}\right)^{0.19} * \exp \left(1.69 *\left(1-\frac{D_{\mathrm{a}}}{D_{\mathrm{r}}}\right)\right) \\
D_{\mathrm{a}}=R^{*} \mathrm{t}_{0} \\
\boldsymbol{N}_{r}=\boldsymbol{a}+\boldsymbol{b} * \boldsymbol{R}+\boldsymbol{C} * \boldsymbol{R}^{2}
\end{gathered}
$$

In the equation, $\mathrm{n}$ is the cycle life of energy storage; $D_{\mathrm{r}}$ is the rated discharge depth, the corresponding number of cycles is $N_{r} ; D_{\mathrm{a}}$ is the depth of discharge under actual conditions; $R$ is the operating rate; $\mathrm{t}_{0}$ is the charging or discharging time; $a, b$, and $c$ are all obtained by fitting the actual operating data.

From equations (1), (2), and (3), the cycle life of energy storage decreases as charge/discharge rate increases. The model can be used to calculate the cycle life of energy storage in different operating modes to determine the number of battery replacements throughout project operation period .

\section{Whole-life-cycle income model of energy storage}

\subsection{Objective function}

The total income of the energy storage is two kinds of services revenue minus its cost. The function aims to maximize the total profit $M$.

$$
\max M=B_{e}+B_{f}-C_{0}
$$

In the equation: $B_{e}$ is the income of time-shift energy arbitrage service ; $B_{\mathrm{f}}$ is the income of frequency regulation service; $C_{0}$ is the whole-life-cycle cost.

\subsubsection{Time-shift energy arbitrage service income}

$$
B_{e}=\left(E_{d} * \sum_{t=1}^{24} P_{d}(t) d t-E_{c} * \sum_{t=1}^{24} P_{c}(t) d t\right) * N * 365
$$

In the equation: $E_{d}$ is the electricity price during discharging period; $E_{\mathrm{c}}$ is the electricity price during charging period; $P_{d}(t)$ and $P_{c}(t)$ are the discharging and charging power of the energy storage at time t.

\subsubsection{Frequency regulation service income}

Based on the market rules of PJM ancillary services, the frequency regulation income consists of two parts: capacity compensation and mileage compensation.

$$
B_{f}=N * 365 * \sum_{t}^{T} P_{r} *\left(E_{f}+E_{p f} * \lambda\right) * K * \Delta \mathrm{t}
$$

In the equation: $\mathrm{P}_{\mathrm{r}}$ is the charge/discharge power of frequency regulation ; $E_{f}$ is the capacity price; $E_{p f}$ is the mileage price; $K$ is the operating performance score; $\lambda$ is the mileage ratio between Reg D and Reg A; $\Delta t$ is the average duration of each frequency regulation; $T$ is total frequency regulation time in one day.

\subsubsection{Whole-life-cycle cost}

The whole-life-cycle cost of energy storage is mainly composed of initial investment cost, replacement cost and maintenance cost. 


$$
C=\frac{C_{e} * Q_{\max }}{\eta}+C_{p} * P_{\max }+C_{\text {rep }}+C_{\text {fom }}
$$

In the equation: $Q_{\max }$ is energy storage rated electricity; $P_{\max }$ is energy storage rated power; $C_{\text {rep }}$ is the cost of battery replacement; $C_{\text {fom }}$ is the operation and maintenance cost; $C_{e}$ is the unit price of lithium ion battery; $\eta$ is the charge and discharge efficiency; $C_{p}$ is the unit price of the power conversion equipment and construction cost.

$$
\begin{gathered}
N_{0}=\frac{365 * m * \mathrm{~N}}{\mathrm{n}} \\
C_{\text {rep }}=\sum_{a=1}^{N_{0}} C_{b a t} *(1+u)^{\frac{N * a}{N_{0}}} *(1-r)^{\frac{N * a}{N_{0}}} \\
C_{\text {fom }}=\sum_{b=1}^{N} C_{e x e} * P_{\max } *(1+u)^{b} *(1-r)^{b}
\end{gathered}
$$

In the equation: $N_{0}$ is the number of battery replacements; $m$ is the charging and discharging times in one day; $u$ is annual reduction rate of battery price; $r$ is the discount rate; $C_{\text {exe }}$ is the unit price of maintenance cost in one year.

\subsection{Restrictions}

\subsubsection{Power constraints}

$$
\left\{\begin{array}{l}
0 \leq P_{d}(t) \leq u_{1} P_{\max } \\
0 \leq P_{c}(t) \leq u_{2} P_{\max } \\
u_{1}+u_{2} \leq 1 \\
u_{1}, u_{2} \in(0,1)
\end{array}\right.
$$

In the equation: $u_{1}$ and $u_{2}$ are $0-1$ variables of discharge and charge power for the corresponding period. These constraints limit that storage energy cannot be charged and discharged at the same time, and the operating power cannot exceed the rated power.

\subsubsection{Electricity constraints}

$$
\begin{gathered}
Q(t)=Q_{0}-\int_{0}^{T}\left(P_{\mathrm{d}}(t)-\frac{P_{\mathrm{c}}(t)}{\eta}\right) d t \\
S(t)=\frac{Q(t)}{Q_{\text {max }}} \\
S_{\text {min }} \leq S(t) \leq S_{\text {max }}
\end{gathered}
$$

In the equation: $Q_{0}$ is the initial electricty of energy storage; $Q(t)$ is the energy storage electricty at time $t ; S(t)$ is the state of charge (SOC) of energy storage at time $t ; S_{\min }$ and $S_{\max }$ are the upper and lower limits of energy storage SOC.

\section{Case analysis}

In this paper, two kind of energy storage operation strategies are proposed. The first strategy is to operate at rated power; the second strategy is to operate at optimal rate, which is the optimization method proposed. 


\subsection{Parameters setting}

The energy storage cluster consists of $500 \mathrm{~kW} / 1000 \mathrm{kWh}(\mathrm{A}), 1000 \mathrm{~kW} / 1000 \mathrm{kWh}$ (B), 2000 $\mathrm{kW} / 1000 \mathrm{kWh}(\mathrm{C}), 4000 \mathrm{~kW} / 1000 \mathrm{kWh}(\mathrm{D})$, and $8000 \mathrm{~kW} / 1000 \mathrm{kWh}(\mathrm{E})$. The project running period N is 10 years. The PJM market data in May 2018 was used for analysis.

\subsection{Calculation results and analysis}

\subsubsection{Economic analysis of time-shift energy arbitrage service}

As shown in Fig. 1, the profit of 1000 kWh energy storage providing time-shift energy arbitrage service will increases first with power then decreases. The reason is that as charge and discharge rate increases, the degradation of battery performance leads to an increase in the number of battery replacements, which in turn leads to an increase in the life cycle cost. After a certain inflection point, the income of time-shift energy arbitrage service exceeds its operating cost, resulting in a decrease in energy storage profit.

In this mode, the optimal power of $1000 \mathrm{kWh}$ energy storage is $1000 \mathrm{~kW}$, and the corresponding optimal operating rate is $1.0 \mathrm{C}$. Therefore, the optimal operating power of energy storage A and B is its rated power; the optimal operating power of energy storage C, D, E is $1000 \mathrm{~kW}$. Taking energy storage $\mathrm{E}$ as an example, the time-shift arbitrage service profit of the energy storage in the rated power mode is $¥$-97.88 million, and the service profit in the optimal power mode is $¥ 1,200,600$. After adopting optimized control strategy, the time-shift energy arbitrage profit of $8000 \mathrm{~kW} / 1000$ kWh turned from negative to positive.

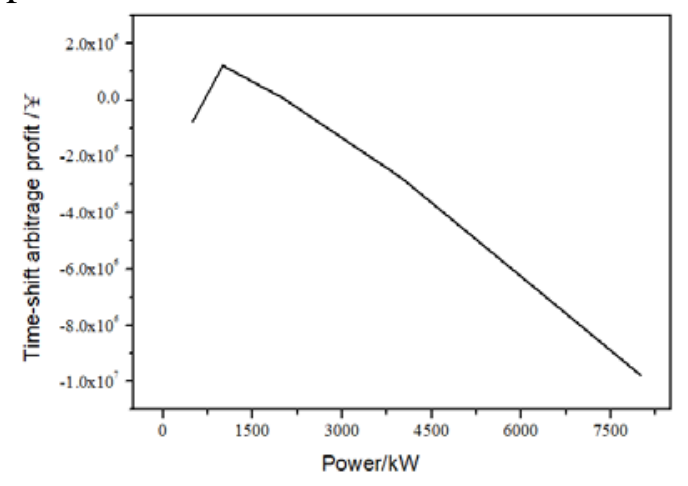

Fig. 1 Relation of the time-shift arbitrage profit and power

\subsubsection{Economic analysis of frequency regulation service}

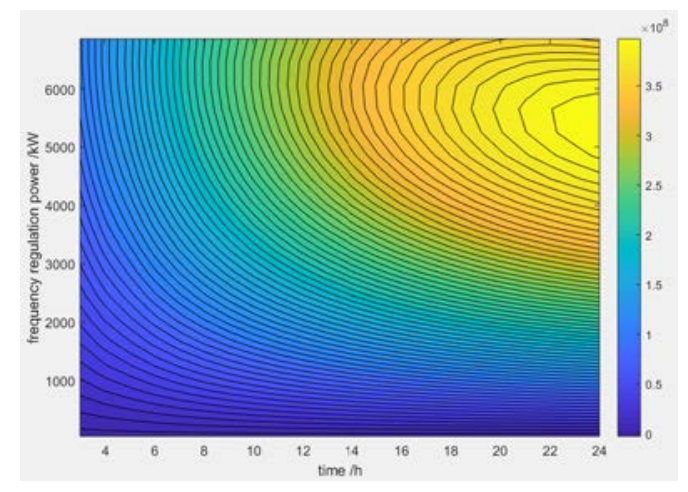

Fig. 2 Relation of the frequency regulation profit and time or power

As shown in Fig. 2, the frequency regulation service profit of $1000 \mathrm{kWh}$ energy storage will increases first and then decreases with the increase of operating power. The optimal operating power is $5384 \mathrm{~kW}$, and the optimization operation strategy of energy storage is to charge/dischage at $5.38 \mathrm{C}$. Therefore, the optimal operating power of energy storage A, B, C, D is its rated power; the optimal operating power of energy storage $\mathrm{E}$ is $5384 \mathrm{~kW}$. Taking energy storage $\mathrm{E}$ as an example, the operating cost of the energy storage in the rated power mode is $¥ 221$ million. In the 
optimal rate mode, the frequency regulation profit is $¥ 396$ million. After the energy storage adopts the optimized operation strategy, its profit of frequency regulation service increased by $79.2 \%$.

\section{Conclusions}

In order to improve economy, energy storage can provide a variety of services. In this paper, considering the factors such as charge/discharge rate and battery life, aiming at maximizing system income, the whole-life-cycle income model and the optimal control strategy are proposed. Applying this model combined with PJM market data, the economy of two kind operation strategies is simulated and analyzed. The main findings are as follows:

According to the energy storage whole-life-cycle income model, the optimal control strategy for energy storage to provide the time-shift energy arbitrage service is to charge/dischage at $1 \mathrm{C}$, and the optimal control strategy for energy storage to provide the frequency regulation service is to charge/dischage at $5.38 \mathrm{C}$.

After adopting optimized control strategy, the time-shift energy arbitrage profit of $8000 \mathrm{~kW} / 1000$ $\mathrm{kWh}$ turned from negative to positive, and its cost-benefit ratio of frequency regulation service increased by $79.2 \%$.

\section{Acknowledgements}

This work is supported by Science and Technology Project of State Grid Jibei Electric Power (Research on typical design of distributed energy storage, No. 52018F170002)

\section{References}

[1] Schoenung S, Hassenzahl W. Long- vs short-term energy storage technologies analysis: a life-cycle cost study[R]. New Mexico and California: Sandia National Laboratories, 2003.

[2] Pudjianto D, Aunedi M, Strabac G. Whole-system assessment of the value of energy storage in low-carbon electricity systems[J]. IEEE Transactions on Smart Grid, 2014, 5(2): 1098-1108.

[3] Dagdougui H, Mary N, Beraud-Sudreau A, et al. Power management strategy for sizing battery system for peak load limiting in a university campus[C]. Smart Energy Grid Engineering (SEGE), 2016 IEEE. IEEE, 2016: 308-312.

[4] LOU Suhua, YI Lin, WU Yaowu, et al. Optimal configuration of battery energy storage capacity based on variable life model[J]. Transactions of China Electrotechnical Society, 2015, 30(4): 265-271.

[5] LI Xinran, LU Longhui, LU Chaoxian, et al. Research on Lithium-ion Battery Capacity Predictive Modeling and Its Simulation[J]. Journal of System Simulation, 2016, 40(8): 2471-2476 